\title{
ON GROUPS WITH SPECIAL ANTIAUTOMORPHISMS
}

\section{HERMANN HEINEKEN}

(Received 13 May 1969)

To Bernhard Hermann Neumann on his 60th birthday

Communicated by G. E. Wall

This note is concerned with the following question: What is the structure of those groups which possess two antiautomorphisms different from identity such that every element of the group is fixed by (at least) one of them?

C. Ayoub [1] stated this problem after having proved a statement equivalent to the following: The group $G$ is a non-abelian extension of an abelian group by a group of order two, if, and only if, there is an automorphism $\alpha \neq 1$ and an antiautomorphism $\beta \neq 1$ such that every element of $G$ is fixed by $\alpha$ or by $\beta$. The Theorem at the end of the paper will show that the class of groups considered by C. Ayoub coincides with the class considered here.

The arguments used here are of a combinatorical nature like those used in the papers by Ayoub [1] and by Haber and Rosenfeld [2].

In the following we consider a group $G$ possessing two antiautomorphisms $\alpha, \beta$ (both different from the identity) such that each element $g$ in $G$ is fixed by at least one of the antiautomorphisms $\alpha, \beta$. We define three subsets $A, B, C$ of $G$ in the following manner:

$$
\begin{aligned}
& C=\left\{x \in G \mid x=x^{\alpha}=x^{\beta}\right\} \\
& B=\left\{x \in G \mid x=x^{\beta} \neq x^{\alpha}\right\} \\
& A=\left\{x \in G \mid x=x^{\alpha} \neq x^{\beta}\right\} .
\end{aligned}
$$

Neither $A$ nor $B$ is empty because the two antiautomorphisms are different from the identity. Every element belongs to the same set as its inverse. The sets $A$ and $B$ may be interchanged in any statement; if we interchange $A$ and $B$ in the statement $(n)$, we will denote the new statement by $\left(n^{\prime}\right)$.

(1) If $x$ and $y$ are in $A \cup C$, then $x y$ is in $A \cup C$ if and only if $x y=y x$.

This follows from $(x y)^{\alpha}=y^{\alpha} x^{\alpha}=y x$ for $x$ and $y$ in $A \cup C$.

(2) $C$ is a commutative subgroup of $G$.

Let $x$ and $y$ be in $C$. Then $x y$ is in $A \cup C$ or $B \cup C$. According to (1) (or (1')) we obtain $x y=y x$ in both cases, and $\left(1^{\prime}\right)$ (or (1)) yields that $x y$ is contained in 
$A \cup C$ and in $B \cup C$. So $x y=y x$ is contained in $C$, which shows that $C$ is a commutative subgroup.

(3) If $x \in A$ and $y \in B, x y \neq y x$.

Assume $x y=y x$, and furthermore, without loss of generality, $x y \in A \cup C$. Now $y=x^{-1}(x y)=(x y) x^{-1} \in A \cup C$ by (1), contrary to hypothesis. This shows (3).

(4) For $x, y \in A$ and $z \in A \cup C$ the following three statements cannot hold simultaneously:

$x y=y x ; \quad z x=x z ; \quad y z \neq z y$.

If the three statements would hold, $y z$ would be contained in $B$ by (1) and would commute with $x \in A$, contrary to (3).

(5) If $z \in C$, either $z \in Z(G)$ or $z$ does not commute with any element not contained in $C$.

Assume the existence of two elements $x, y$ which are not contained in $C$ such that $x z=x z$ but $y z \neq z y$. Without loss of generality we may assume that $x$ and $y$ are both elements of $A$ (if $x$ and $y$ do not belong to the same set, $x$ and $y z$ do so according to (1) or $\left(1^{\prime}\right)$, and we take $y z$ instead of $\left.y\right)$. By (4) we know that $x y \neq$ $y x$; furthermore $(x y) z \neq z(x y)$. Now $x y \in B$ by (1) and $z(x y) \in A$ by $\left(1^{\prime}\right)$. It follows from (1) and (2) that $z x=x z$ belongs to $A$ and that $z x$ commutes with $y$, because $z x, y$ and $(z x) y$ are contained in $A$. Now $z x$ commutes with $x$ and $y$ while $x$ does not commute with $y$. This contradiction to (4) proves (5).

(6) $C=Z(G)$, or $Z(G)$ is a subgroup of index two in $C$.

By (3) we obtain $Z(G) \subseteq C$. Assume now that $Z(G) \neq C$ and choose two elements $u, v$ of $C$ which do not belong to $Z(G)$. If $a$ is any element in $A, a u \in B$ by (5) and (1), further $(a u) v \in A$ by (5) and (1'). So, by (1), $a(u v)=(u v) a$, and $u v \in Z(G)$ by (5). This proves that $Z(G)$ is of index two in $G$.

(7) If $x$ and $y$ belong to $G$ but not to $Z(G)$, then $C(x)=C(y)$ or $C(x) \cap C(y)=$ $Z(G)$, where $C(u)$ is the centralizer of $u$ in $G$.

If $x$ or $y$ is contained in $C$, (7) is true by (5). If both $x$ and $y$ are not contained in $C$ and if they do not belong to the same set $A, B$, then $C(x) \cap C(y) \subseteq C$ by (3), so $C(x) \cap C(y)=Z(G)$ by (5). If both the elements $x, y$ belong to $A$ (or to $B$ ) and $C(x) \neq C(y)$, the intersection is contained in $C$ by (4), so $C(x) \cap C(y)=Z(G)$ by (5).

(8) If $x, y \in A$ and $x^{2} y \neq y x^{2}$, then $y^{2} \in Z(G)$.

By (1), $x y$ and $x^{2} y$ are contained in $B$. As $x$ does not commute with $y$ and $x y$, likewise $x y$ does not commute with $x^{2} y$. So $y^{-1} x y=(x y)^{-1}\left(x^{2} y\right)$ belongs to $A$ by $\left(1^{\prime}\right)$. Now 


$$
\begin{aligned}
y^{-1} x y & =\left(y^{-1} x y\right)^{\alpha}=y^{\alpha} x^{\alpha}\left(y^{-1}\right)^{\alpha}=y x y^{-1} ; \\
x y^{2} & =y^{2} x .
\end{aligned}
$$

Hence $y^{2} \in C(x) \cap C(y)=Z(G)$ because $x \notin C(y)$, using (7).

(9) $G / Z(G)$ possesses elements of order two.

Easy consequence of (8).

According to (7), the quotient group $G / Z(G)$ possesses a partition formed by the subgroups $C(x) / Z(G), x \notin Z(G)$. We consider first of all groups possessing not more than four different centralizers.

(10) If $G$ does not possess more than four different centralizers of elements which are proper subgroups, then either

(a) $G / Z(G)$ is isomorphic to the elementary abelian group of order four, or

(b) $G / Z(G)$ is isomorphic to the symmetric group on three objects.

Groups covered by three subgroups possess a normal subgroup such that the corresponding quotient group is elementary abelian of order four, see Haber and Rosenfeld [2; Theorem 2, p. 492]. It follows that groups partitioned by three subgroups must be elementary abelian and of order four. Similarly we obtain that groups partitioned by four subgroups are either elementary abelian and of order 9, or isomorphic to $S_{3}$ (see [2; Theorem 4, p. 494]). The case that $G / Z(G)$ is an elementary abelian group of order 9 is excluded by (9), so (10) is a complete characterization.

If $G / Z(G)$ is partitioned by five or more centralizers, we may assume (without loss of generality) that there are three or more different centralizers which are contained in $A \cup C$. It is our aim to show that $B \cup Z(G)$ is an abelian subgroup of index two in $G$. We collect all the necessary steps in

(11) Assume that there are three elements $x, y, z$ in $A \cup C$ which do not commute with each other. Then

(a) $C(x y)=C\left(x y^{-1}\right)=C\left(x^{-1} y\right)=C(y x)=C(y z)$,

(b) If $c d=d c$, where $c$ and d belong to $A \cup C$ but not to $Z(G)$, then $c d \in Z(G)$.

(c) If $t, u, v, w \in A \cup C$ and $t u, v w \in B$, then $(t u)(v w)=(v w)(t u)$.

(d) If $u, v, w$ are contained in $A \cup C$ but not in $Z(G)$, the same holds for uvw.

(e) If $s, t \in B$ then $s t=t s$.

(f) $B \cup Z(G)$ is an abelian subgroup of index 2 in $G$.

By (1), $y x, x^{-1} z$ and $y z=(y x)\left(x^{-1} z\right)$ are contained in $B$.

Using $\left(1^{\prime}\right)$ and $(7)$ we find

$$
C(y z)=C(y x)=C\left(x^{-1} z\right),
$$

and, by similar procedure, 


$$
\begin{aligned}
C\left(x^{-1} z\right) & =C\left(x^{-1} y\right)=C\left(y^{-1} z\right), \\
C(y z) & =C\left(y x^{-1}\right)=C(x z) \\
C(x z) & =C(x y)=C\left(y^{-1} z\right) .
\end{aligned}
$$

Now (a) is a consequence of these equations.

To prove (b), assume that $c$ and $d$ do not commute with $y$ and $z$. We obtain from (a) for $c, y, z$

and for $d, y, z$

$$
c y^{-1} \in C\left(c y^{-1}\right)=C(y z)
$$

$$
y d \in C(y d)=C(y z) .
$$

Now $c d=\left(c y^{-1}(y d) \in C(y z) \cap(A \cup C)=Z(G)\right.$, since $y z \in B$.

By assumption in (c), $t$ does not commute with $u$ and $v$ does not commute with $w$. Assume, for instance, that $t$ commutes with $v$. Then $v^{-1} t$ belongs to $Z(G)$ by (b), and $t=v a$ with $a \in Z(G)$. So $C(t u)=C(v a u)=C(v u)$. Using this process of reduction, we prove (c) by (a), looking at each possibility of commuting pairs of elements.

$$
\begin{aligned}
& C(v w)=C(t u) \quad \text { if } t v=v t \text { and } u w=w u \\
& C(v w)=C(u t)=C(t u) \text { if } t w=w t \text { and } u v=v u \\
& C(v w)=C(u w)=C(t u) \text { if } u v=v u \text { and } t w \neq w t \\
& C(v w)=C(v u)=C(t u) \text { if } u w=w u \text { and } t v \neq v t \\
& C(v w)=C(t w)=C(t u) \text { if } v t=t v \text { and } u w \neq w u \\
& C(v w)=C(v t)=C(t u) \text { if } w t=t w \text { and } u v \neq v u \\
& C(v w)=C(w t)=C(t u) \text { if no pairs commute. }
\end{aligned}
$$

If in (d) any two elements of the triplet $u, v, w$ commute, we find $u v w=w v u \in A \cup C$ by (b), and it is easy to show with (b) that $u v w$ is not contained in $Z(G)$. If $u, v, w$ do not commute with each other, we obtain from (c)

$$
(v u)(v w)=(v w)(v u) \text {, so } u v w=w v u,
$$

and $u v w \in A \cup C$. If $u v w$ is contained in $Z(G)$, then

$$
u v=(u v w) w^{-1} \in A \cup C,
$$

but $u v \in B$ because $u$ and $v$ do not commute. This shows (d).

Assume now that there are elements in $B$ which do not commute with each other. Then, by (7), there is an element $b$ in $B$ which does not commute with $x y$, where $x$ and $y$ are the elements mentioned at the beginning of (11). Then $b x y \in A$, and there exists an element $w$ in $A \cup C$ which does not commute with $b x y$. Hence $(b x y) w$ belongs to $B$, and $x y w$ belongs to $A \cup C$ but not to $Z(G)$ according to (d). Using (d) again we find that $(b x y) w(x y w)^{-1}$ is contained in $A \cup C$. But (bxy)w(xyw) $)^{-1}=b \in B$; a contradiction establishing (e).

If $b$ is any element in $B, C(b)$ is a commutative subgroup of $G$ by (4), $C(b) \cup A$ 
is empty by (3), $B \subset C(b)$ by (11e) and $C \cap C(b)=Z(G)$ by (5). So $C(b)=$ $B \cup Z(G)$ is an abelian subgroup of $G$. Assume that $c$ and $d$ are two elements of $G$ which are not contained in $B \cup Z(G)$. Then they are contained in $A \cup C$ but not in $Z(G)$, and their product $c d$ is contained in $B$ if they do not commute (by 1$)$ ); if they commute the product is contained in $Z(G)$ by $(11 \mathrm{~b})$. So the product of any two elements outside $B \cup Z(G)$ belongs to $B \cup Z(G)$, hence $B \cup Z(G)$ is of index two in $G$; the proof of (11) is complete.

Taking the statements (10) and (11) together we have obtained that $G$ possesses an abelian subgroup $N$ of index two in $G$, also $G$ is non-abelian (see (6)). We will now construct the two antiautomorphisms to show that all these groups occur.

Let $N$ be an abelian normal subgroup of the non-abelian group $G$ such that $G=\{x, N\}$ and $x^{2} \in N$. We take $\alpha$ such that $(x n)^{\alpha}=x n$ for all $n \in N$. If $\alpha$ is an antiautomorphism, we find

consequently

$$
x n=(x n)^{\alpha}=n^{\alpha} x^{\alpha}=n^{\alpha} x,
$$

$$
n^{\alpha}=x n x^{-1},
$$

and the mapping defined in this way is indeed an antiautomorphism, as can be checked by the reader. On the second mapping $\beta$ we impose $n^{\beta}=n$ for all $n \in N$. Furthermore we put $x^{\beta}=x u$ for some $u \in N$.

For $\beta$ to be an antiautomorphism it is necessary, that

which is equivalent to

$$
x^{2}=\left(x^{2}\right)^{\beta}=(x u)^{2},
$$

$$
x^{-1} u x=u^{-1} .
$$

The reader will be able to check that $\beta$ is an antiautomorphism if it is defined by

$$
n^{\beta}=n \quad \text { for all } n \in N
$$

and

$$
(x n)^{\beta}=x u\left(x^{-1} n x\right) \text { for all } n \in N,
$$

provided (*) is satisfied by $u$.

We summarize:

THEOREM: The following properties of the group $G$ are equivalent:

(i) $G$ is non-abelian and possesses an abelian normal subgroup of index two.

(ii) $G$ possesses an automorphism $\sigma \neq 1$ and an antiautomorphism $\rho \neq 1$ such that for all $x \in G$ either $x^{\sigma}=x$ or $x^{\sigma}=x^{\rho}$ is true.

(iii) $G$ possesses a proper subgroup $U$ and an antiautomorphism $\gamma \neq 1$ such that all elements of $G$ which are not contained in $U$ are fixed by $\gamma$.

(iv) G possesses an antiautomorphism $\beta \neq 1$ and an automorphism $\alpha \neq 1$ such that for all $x \in G$ either $x^{x}=x$ or $x^{\beta}=x$. 
(v) G possesses two antiautomorphisms different from identity such that all $x \in G$ are fixed by at least one of them.

Proof. The equivalence of (i) and (v) is the contents of the preceding argument. The statements (i) and (ii) are equivalent by Theorem 1 and Theorem 2 of Ayoub [1]. To see that (iv) follows from (ii), take $\sigma=\alpha$ and $\rho \sigma^{-1}=\beta$; for the converse take $\alpha=\sigma$ and $\beta \alpha=\rho$. If we remember that the set of elements fixed by an automorphism is a subgroup it is easy to obtain (iii) from (iv). Assume now that (iii) is true, and assume that $x \in G$ is not contained in $U$. By (iii) $x u=$ $(x u)^{y}$ for all $u \in U$, and we obtain

$$
\begin{aligned}
x u & =u^{\gamma} x^{\gamma}=u^{\gamma} x ; \\
u^{\gamma} & =x u x^{-1} \\
x\left(u_{1} u_{2}\right) x^{-1} & =\left(u_{1} u_{2}\right)^{\gamma}=u_{2}^{\gamma} u_{1}^{\gamma}=\left(x u_{2} x^{-1}\right)\left(x u_{1} x^{-1}\right)=x\left(u_{2} u_{1}\right) x^{-1}
\end{aligned}
$$

for all $u_{1}, u_{2} \in U$.

We conclude that $U$ is abelian. The group $G$ cannot be abelian, for otherwise $G$ would be the set-theoretical union of the subgroup $U$ and the subgroup consisting of all elements fixed under $\gamma$, which is impossible. Every subgroup of $G$ containing $U$ is fixed as a subgroup under $\gamma$, so by the preceding argument $U$ is maximal with respect to being abelian. Assume now the existence of an element $y \in G$ such that $U \neq y U \neq x U$. Then

$$
(y u)^{\gamma}=y u \text { yields } u^{y}=y u y^{-1} .
$$

But

$$
u^{\gamma}=x u x^{-1},
$$

so

$$
y^{-1} x \in C(U)=U,
$$

contrary to $x U \neq y U$.

This shows that $U$ is of index two in $G$, so all the statements of (i) are obtained trom (iii) The proof is complete.

I am indebted to Dr. E. Wittmann for his many helpful remarks concerning this problem.

\section{References}

[1] C. Ayoub, 'Constructing an automorphism from an antiautomorphism', Can. Math. Bull. 11 (1968), 367-370.

[2] S. Haber and A. Rosenfeld, 'Groups as unions of proper subgroups', Amer. Math. Monthly 66 (1959), 491-494.

Universität Erlangen-Nürnberg

852 Erlangen

West-Germany 